\title{
The effect of dietary changes on distinct components of the metabolic syndrome in a young Sri Lankan population at high risk of CVD
}

\author{
Nicola Guess ${ }^{1 *}$, Mahen Wijesuriya ${ }^{2}$, Laksha Vasantharajah ${ }^{2}$, Martin Gulliford ${ }^{3}$, Giancarlo Viberti ${ }^{4}$, \\ Luigi Gnudi ${ }^{4}$ and Janaka Karalliedde ${ }^{4}$ \\ ${ }^{1}$ Department of Medicine, Imperial College London, London W12 ONN, UK \\ ${ }^{2}$ Diabetes Association of Sri Lanka, Colombo, Sri Lanka \\ ${ }^{3}$ King's College London, Primary Care \& Public Health Sciences, London SE1 1UL, UK \\ ${ }^{4}$ Cardiovascular Division, King's College London, London SE1 9NH, UK \\ (Submitted 10 February 2016 - Final revision received 4 May 2016 - Accepted 23 May 2016 - First published online 30 June 2016)
}

\section{Abstract}

South Asian populations are predisposed to early onset of the metabolic syndrome. Lifestyle intervention programmes have demonstrated a reduction in the metabolic syndrome and CVD risk; however, the most effective components of the multi-faceted lifestyle interventions are unknown. We studied 2637 Sri Lankan males ( $n$ 1237) and females ( $n 1380$ ), with a mean BMI of $23 \cdot 9$ (sD $4 \cdot 2$ ) kg/m², aged $22 \cdot 5$ (sD $10 \cdot 0)$ years, who had participated in a 5-year lifestyle-modification programme to examine the effect of dietary changes on distinct components of the metabolic syndrome. The dietary intervention comprised advice to replace polished starches with unpolished starches, high-fat meat and dairy products with low-fat products and high-sugar beverages and snacks with low-sugar varieties. For the purposes of this analysis, data from the control and intensive lifestyle groups were combined. Anthropometric and biochemical data were recorded, and a FFQ was completed annually. Multiple regression was used to determine the effect of the dietary changes on distinct components of the metabolic syndrome. The ratio unpolished:polished rice was inversely related to change in fasting glucose $(\beta=-0.084, P=0.007)$ and TAG $(\beta=-0.084, P=0.005)$ and positively associated with change in HDL-cholesterol $(\beta=0.066, P=0.031)$ at the 5 -year follow-up after controlling for relevant confounders. Red meat intake was positively associated with fasting glucose concentrations $(\beta=0 \cdot 05, P=0 \cdot 017)$, whereas low-fat $(\beta=-0 \cdot 046, P=0 \cdot 018)$ but not high-fat dairy products $(\beta=0.003, P=0.853)$ was inversely related to glucose tolerance at the follow-up visit. Replacement of polished with unpolished rice may be a particularly effective dietary advice in this and similar populations.

\section{Key words: Dietary fibres: Glycaemic index: Whole grains: Dairy products: Fasting glucose}

The metabolic syndrome (MetS) constitutes a cluster of cardiovascular risk factors, which double the risk for $\mathrm{CHD}$ and increase the risk for type 2 diabetes mellitus (T2DM) 5-fold ${ }^{(1)}$. Although the MetS is a growing epidemic worldwide, the prevalence reaches $25-50 \%$ in some urban areas of South Asia, and the prevalence of individual risk factors is even greater, with obesity and low HDL-cholesterol reaching 68 and $81 \%$, respectively ${ }^{(2)}$. These risk factors are also growing in prevalence among children and young adults ${ }^{(2)}$.

The risk of the MetS can be reduced through diet and lifestyle changes ${ }^{(3,4)}$. In particular, approaches including weight loss ${ }^{(4,5)}$, increasing whole-grain sources of carbohydrates ${ }^{(6,7)}$ and increasing dairy products intake ${ }^{(8)}$ have a demonstrable effect on components of the MetS. However, the majority of studies on the MetS have been carried out in older, Western populations or Southern Asians who have migrated to Western countries ${ }^{(4-9)}$.

There are differences in the presentation of the MetS in Southern Asians, who are at higher risk of MetS risk factors such as increased waist circumference (WC) and hyperglycaemia at a given BMI along with lower HDL-cholesterol and higher levels of small, dense LDL-cholesterol compared with their Caucasian counterparts $^{(2)}$. Diet and lifestyle factors may also differ - for example, Indians obtain a higher percentage of their energy content from carbohydrates compared with Europeans, potentially increasing the risk of hypertriacylglycerolaemia ${ }^{(10)}$. Lower intakes of MUFA, $n$-3 PUFA and fibre and higher intakes of total fat, SFA and trans-fat have also been reported ${ }^{(11)}$.

The effect of small changes in diet on metabolic risk factors is subtle and occurs over time. Therefore, prospective studies are needed to account for confounding variables such as weight change and longitudinal trends in dietary intake. Furthermore, it is important to note that a-one-size-fits all approach may not be appropriate given the heterogeneous presentation of the MetS. For example, a recent study found that a $10 \%$ increase in the consumption of rice was associated with lower weight gain, reduced risk of hypertension, but an increased risk of

Abbreviations: 2hPG, 2-h plasma glucose; FPG, fasting plasma glucose; MetS, metabolic syndrome; T2DM, type 2 diabetes mellitus; WC, waist circumference.

* Corresponding author: Dr N. Guess, fax +44 208383 8320, email n.guess10@imperial.ac.uk 
hyperglycaemia ${ }^{(12)}$, indicating that diets could be tailored to the prevalent risk factors. Studies with large sample sizes are needed to examine the effects of specific nutrients on components of the MetS ${ }^{(12-14)}$

The DIABRISK-SL study ${ }^{(15)}$ is a randomised controlled parallel group clinical trial performed at a single centre in Colombo, Sri Lanka, to examine the effects of a lifestyle intervention on the primary composite cardio-metabolic end points of new-onset T2DM, impaired glucose tolerance, impaired fasting glycaemia, new-onset hypertension and albuminuria, following 5 years of intervention. In this study, we explore the dietary factors that mediate the beneficial effects on the primary outcomes.

\section{Methods}

The design of the trial is described in detail elsewhere ${ }^{(15)}$. In brief, 4683 Sri Lankan urban young males and females aged 5-40 years at high risk of the MetS were randomised to an intensive lifestyle-modification group or to a less-intensive control group. The representative sample was recruited following screening of 23298 individuals aged 5-40 years in schools, workplaces, universities and community organisations across Columbo, Sri Lanka. Clinical and dietary data were collected at baseline and annually up to the final follow-up. In this study, we utilised only the data of the 2637 subjects who completed the 5-year study and for whom complete dietary data were available. All participants provided written informed consent, and the study was given ethics approval by the Sri Lanka Medical Association Ethical Review Committee (ERC 07-010). Permission from the Ministry of Education was obtained for this study and was conducted under the Good Clinical Practice Guidelines and according to the principles expressed in the Declaration of Helsinki for clinical research.

\section{Participants}

Inclusion criteria were aged 5-40 years with two or more of the following risk factors for cardio-metabolic disease: first-degree family history of T2DM, physical inactivity, elevated BMI and raised WC. These risk factors were defined as follows: physical inactivity ( $<30 \mathrm{~min}$ continuous exercise for $<3 \mathrm{~d} /$ week); WC (in subjects between 5 and 17 years defined as $\geq 91$ th percentile; 18-40 years: females $\geq 80 \mathrm{~cm}$ and males $\geq 90 \mathrm{~cm}$ ); and raised BMI (in subjects aged 5-18 years defined as a BMI value greater than internationally standardised age- and sex-specific percentile cut-offs and between 18 and 40 years as BMI $\left.\geq 23 \mathrm{~kg} / \mathrm{m}^{2}\right)^{(15)}$. Exclusion criteria included subjects with no or only one identifiable risk factor; subjects with diagnosed end points (T2DM, CVD, hypertension and renal disease); or subjects on any form of medication used for the treatment of diabetes, hypertension, renal disease or dyslipidaemia either at screening or during the study. Subjects were also excluded if they had any active communicable or non-communicative diseases such as cancer, asthma or other forms of chronic lung disease, depression, tuberculosis or were or became pregnant either at screening or during the study.

\section{Intensive v. control group}

Subjects were randomised to an intensive lifestyle intervention group in which they had three monthly telephone and/or face-to-face contacts to assess progress and reiterate goals. The total number of one-to-one contacts was four per year. In the control group, the same advice was given, but with the face-to-face contacts being annual. For the purposes of this analysis, the intervention and control groups were combined.

\section{Dietary goals}

Overall, lifestyle advice was based on the Indian Diabetes Prevention Program ${ }^{(16)}$ : (1) individual advice to balance food intake and physical activity and to achieve or maintain appropriate body weight; (2) avoidance of simple sugars and refined carbohydrates; (3) reduce total fat intake (not to exceed $20 \mathrm{~g} / \mathrm{d}$ ) and restrict the use of SFA; and (4) include more fibre-rich foods such as whole grains, legumes, vegetables and fruits ${ }^{(16)}$. The advice was based on the needs of the subjects. For example, in adults with a raised BMI or WC, advice was given to achieve $5 \%$ weight loss. For children, the aim was to limit weight gain. Subjects were also advised on the importance of regular meals and to avoid delaying or missing meals.

Details on physical activity are described in detail elsewhere ${ }^{(12)}$.

In order to standardise both provision of dietary advice and collection of dietary data, an exchange system was designed to replace energy-dense, high-fat, refined foods (unrecommended items) with low-energy dense, fibre-rich food sources (recommended items) (online Supplementary material) to correspond with the four dietary aims. With the use of the exchange model, participants were guided towards replacing the nonrecommended with recommended items. Although the advice given was individualised, the focus was largely on replacing polished starches with unpolished starches, starchy vegetables with-non starch vegetables, high-fat meats and dairy products with low-fat meats and dairy products and reducing the consumption of sugar-sweetened beverages.

A FFQ was then adapted from the exchange model to determine the number of servings of each food group to allow annual dietary data collection. Serving sizes used were rice serving spoons and tablespoons, and the food recall was completed by a trained peer-to-peer educator with the participant. This exchange model approach was used as it facilitated the provision of effective dietary advice across a population by non-medically or nutritionally trained individuals from local communities, and would therefore be straightforward to translate to similar settings.

The intervention also addressed meal timings such as reducing snacks and consuming regular meals, based on traditional Sri Lankan meals and customs. The number of meals skipped and snacks consumed per day was also recorded by the educator.

\section{Peer-to-peer educators}

The intervention was delivered by peer-to-peer educators recruited from the same communities as the study participants. More than $50 \%$ of the participants were $<18$ years of age, and 
this type of community-driven intervention is an effective approach for promoting lifestyle changes ${ }^{(17)}$. All educators received initial and ongoing training by a team of local and international educators, assessed by the International Diabetes Federation, and supervised by academics from King's College London. A training manual was provided; during weekly meetings throughout the study period, cases were discussed and agreed, and each educator was required to make a weekly presentation on a relevant topic to the principal investigator and head of research. This ensured that correct advice was being imparted to the participants.

\section{Outcomes}

The clinical outcomes are defined in a description of the trial published elsewhere ${ }^{(15)}$. In brief, the MetS was defined by the International Diabetes Federation ${ }^{(14)}$. At baseline and each year of the trial (Y1, Y2, Y3 and Y4), the following outcomes were measured: weight, WC, BMI, fasting plasma glucose (FPG), fasting TAG, fasting HDL-cholesterol and LDL-cholesterol, and blood pressure. At Y3, but not Y4 (final visit), an oral glucose tolerance test (OGTT) was performed. For changes in 2-h plasma glucose (2hPG) as the dependent variable, all other variables entered in the regression equation were also taken from Y3. Physical activity was measured by the International Physical Activity Questionnaire, and dietary intake was self-reported using the culturally appropriate food FFQ.

\section{Statistics}

Exploratory analysis using correlations, independent $t$ tests and one-way ANOVA was carried out to determine variables of interest and inform the regression analyses. Multiple regression analysis was performed to examine the effect of the independent variables of dietary factors on the dependent variables of change in clinical outcomes from baseline to Y4. As the primary advice was to replace non-recommended items with recommended food items, for the independent variables, we created ratios of total recommended items:non-recommended items for each year of data collection, which were averaged from baseline to the final Y4 follow-up. We also created ratios of unpolished:polished rice and low-fat:high-fat food items, which were also averaged over 5 years to account for any changes in diet over the course of the study. We also created a variable for total unpolished:polished starches to include bread, and all associations were the same as for unpolished:polished rice, and thus the data are not presented here. In addition, based on the current literature on dairy products (total, low-fat only and fullfat only) $)^{(8)}$ and soluble fibres (dahl and pulses) ${ }^{(18)}$, we also created two groups for these, again averaged over the study period. We also created averages of weekly intake of specific food groups over the 5-year study period, again based on existing literature - namely, red meat ${ }^{(9)}$, sugar-sweetened beverages $^{(19)}$ and processed meat ${ }^{(20)}$. The dependent variables were the changes from baseline to Y4 follow-up in FPG, HDL-cholesterol, LDL-cholesterol, TAG, WC, blood pressure and weight. Regression with change in weight and WC as outcomes of interest included baseline BMI, the baseline value for dependent variables of interest, sex, age, ethnicity, alcohol intake, smoking status and physical activity (total metabolic equivalents per week averaged over 5 years), and the two major dietary components - unpolished:unpolished rice and non-fat: high-fat food products. The regression models for all other outcome variables were additionally adjusted for change in body weight. An OGTT was performed at Y3, not Y4, so that the effect of dietary variables on 2hPG could be assessed using ratio/food group intake and physical activity averaged over 4 years and weight change upto 4 years, instead of 5 years. Normality tests were performed for all dependent variables and non-normal variables were transformed before analysis; data were checked for homoscedasticity and collinearity of independent variables. Dummy variables were created for categorical independent variables. Standardised $\beta$ values are reported to enable comparison of dietary factors with clinical outcomes. Statistical analyses were performed using SPSS statistical software for Windows, version 14.0 (SPSS). A $P$ value of $<0.05$ was considered to be significant.

\section{Results}

\section{Study participants}

Baseline demographic characteristics and food intake data of all participants with complete dietary data ( $n$ 2637) are shown in Table 1 separated by age ( $>$ or $<18$ years).

\section{Weight and waist change}

All food patterns were significantly related to weight change when examined by the $t$ test. Participants who ate meals outside home $(P<0.0001)$, did not have regular meals $(P<0.0001)$, skipped breakfast $(P<0.0001)$, lunch $(P<0.001)$ or dinner $(P<0 \cdot 001)$, and had $\geq 1$ snacks/d $(P<0 \cdot 0001)$ had significantly higher weight gain at the Y4 follow-up. WC at Y4 was also significantly higher in those who ate meals outside the home $(P<0.0001)$, those who did not eat regular meals $(P<0.001)$, those who skipped breakfast $(P<0.0001)$ and those who had $\geq 1$ snacks/d ( $P<0 \cdot 001)$ (Fig. 1). However, when controlled for age, sex, BMI, physical activity, alcohol intake, baseline weight or WC, only having regular meals was significantly related to weight change $(\beta=-0.037, P=0.032)$ and WC $(\beta=-0.038$, $P=0 \cdot 011)$.

There was no effect of any food or food group on weight change and WC at the Y4 follow-up after controlling for confounders.

\section{Biochemical outcomes}

Fasting plasma glucose. The ratio of total recommended:nonrecommended items $(\beta=-0.049, P=0.023)$ and unpolished: polished rice $(\beta=-0.084, P=0.007)$ was inversely related to change in FPG at Y4 after controlling for age, sex, BMI, physical activity, alcohol intake, baseline FPG and weight change (Table 2) (Fig. 2(a)). Red meat was also positively related to FPG $(\beta=0.05$, $P=0.018$ ), but processed meat as a separate category was not. Not having regular meals was also associated with a higher FPG at the Y4 follow-up $(\beta=0 \cdot 049, P=0 \cdot 01)$ (Table 2$)$. 
Table 1. Baseline demographic characteristics and food intake data (Mean values and standard deviations for demographic variables; medians and interquartile ranges (IQR) for food intake data, which were not normally distributed)*

\begin{tabular}{|c|c|c|c|c|}
\hline \multirow[b]{2}{*}{ Demographic characteristics } & \multicolumn{2}{|c|}{$\begin{array}{l}\text { Under } 18 \text { years } \\
(n 1250)\end{array}$} & \multicolumn{2}{|c|}{$\begin{array}{l}\text { Over } 18 \text { years } \\
\quad(n 1387)\end{array}$} \\
\hline & Mean & SD & Mean & SD \\
\hline Age (years) & $13 \cdot 1$ & $2 \cdot 1$ & 30.9 & $6 \cdot 2$ \\
\hline Sex $(M: F)(\%)$ & \multicolumn{2}{|c|}{$41: 59$} & \multicolumn{2}{|c|}{$54: 46$} \\
\hline Waist $(\mathrm{cm})$ & $79 \cdot 2$ & 11.0 & $87 \cdot 7$ & $9 \cdot 5$ \\
\hline Weight (kg) & $55 \cdot 2$ & $13 \cdot 6$ & $67 \cdot 0$ & $12 \cdot 5$ \\
\hline BMI $\left(\mathrm{kg} / \mathrm{m}^{2}\right)$ & $22 \cdot 5$ & $4 \cdot 2$ & $25 \cdot 1$ & 3.7 \\
\hline $\mathrm{FPG}(\mathrm{mmol} / \mathrm{l})$ & 4.9 & 0.4 & $5 \cdot 0$ & 0.5 \\
\hline 2hPG (mmol/l) & $5 \cdot 8$ & $1 \cdot 1$ & $6 \cdot 0$ & 1.7 \\
\hline $\mathrm{TC}(\mathrm{mmol} / \mathrm{l})$ & 4.6 & 0.8 & $5 \cdot 2$ & 0.8 \\
\hline LDL-cholesterol (mmol/l) & $3 \cdot 5$ & 0.7 & $3 \cdot 4$ & 0.8 \\
\hline HDL-cholesterol (mmol/l) & $1 \cdot 2$ & 0.3 & $1 \cdot 1$ & 0.2 \\
\hline TAG $(\mathrm{mmol} / \mathrm{l})$ & $1 \cdot 1$ & 0.5 & $1 \cdot 3$ & 0.7 \\
\hline Food group (servings/week) & Median & IQR & Median & IQR \\
\hline Brown rice & 6 & 14 & 6 & 14 \\
\hline Pulses & 1 & 2 & 0 & 1 \\
\hline White rice & 7 & 14 & 7 & 14 \\
\hline White bread & 2 & 7 & 1 & 3 \\
\hline Wheat flour products & 0 & 1 & 0 & 1 \\
\hline Green leafy vegetables & 7 & 4 & 6 & 4 \\
\hline Starchy vegetables & 1 & 2 & 1 & 3 \\
\hline Fruity vegetables & 14 & 7 & 21 & 7 \\
\hline Dhal & 7 & 4 & 7 & 4 \\
\hline Sweet fruit & 7 & 4 & 4 & 5 \\
\hline Dates, plums, prunes & 0 & 0 & 5 & 0 \\
\hline Whole milk & 7 & 7 & 7 & 11 \\
\hline Whole milk products & 1 & 3 & 0 & 2 \\
\hline Fresh fish & 7 & 4 & 7 & 3 \\
\hline Chicken & 1 & 2 & 1 & 2 \\
\hline Processed meat & 0 & 2 & 0 & 0 \\
\hline Egg white & 2 & 2 & 2 & 2 \\
\hline Egg yolk & 2 & 2 & 1 & 2 \\
\hline Crisps/chips & 0 & 3 & 0 & 2 \\
\hline Fried rice & 0 & 1 & 0 & 2 \\
\hline Sweetened tea/coffee & 3 & 7 & 7 & 14 \\
\hline Ice cream & 0 & 1 & 0 & 1 \\
\hline Biscuits & 7 & 7 & 2 & 7 \\
\hline Sweetened fruit juice & 0 & 2 & 0 & 0 \\
\hline
\end{tabular}

M, male; F, female; FPG, fasting plasma glucose; 2hPG, 2-h plasma glucose; TC, total cholesterol

* Where median and IQR are $<1$ serving/week, data are not shown.

2- $h$ Plasma glucose. The total amount of recommended: non-recommended food items was not related to $2 \mathrm{hPG}$ at Y4, nor was unpolished:polished rice and low-fat:high-fat diet. However, low-fat dairy products $(\beta=-0 \cdot 046, P=0 \cdot 018)$, but not total or high-fat dairy products $(\beta=0.003, P=0.853 ; \beta=-0.010$, $P=0.601$ ), was inversely related to $2 \mathrm{hPG}$ at the Y4 follow-up (Table 2). Physical activity was also negatively related to $2 \mathrm{hPG}$ (Table 2).

$H D L$-cholesterol. The ratio of total recommended:nonrecommended items $(\beta=0.044, P=0.032)$ and unpolished: polished rice $(\beta=0.066, P=0.031)$ was positively related to HDL-cholesterol concentrations (Table 3) (Fig. 2(b)). There was no relationship between any other dietary variable including pulses or meal patterns and HDL-cholesterol concentrations.
TAG. The ratio of unpolished:polished rice $(\beta=-0.084$, $P=0.005)$, white fish intake $(\beta=-0.059, P=0.003)$ and soluble fibre intake $(\beta=-0.044, P=0.025)$ were also negatively related to TAG concentrations (Table 3 ).

Blood pressure. After adjusting for age, sex, BMI, physical activity, alcohol intake and baseline blood pressure, there was no relationship between meal timings and blood pressure. Total dairy products demonstrated a borderline association with diastolic $(\beta=-0.031, P=0.052)$ blood pressure. When low-fat dairy products were analysed separately, the relationship was significant $(\beta=-0.046, P=0.004)$. There was no relationship between any other dietary variables including fruits and vegetables, low-fat products in general and unpolished: polished rice and blood pressure.

Total and LDL-cholesterol. There was no relationship between any dietary variable and total or LDL-cholesterol.

\section{Relationship between meal timings and food group intake}

Participants who skipped meals had a significantly lower ratio of recommended:non-recommended food items $(P<0.001)$, low:high-fat products $(P<0.0001)$ and unpolished:polished rice $(P<0 \cdot 0001)$ compared with those who did not skip meals. Participants who snacked had a lower ratio of recommended: non-recommended food items $(P<0.0001)$ and low:fat products $(P<0 \cdot 0001)$. Participants who ate meals out of home had a lower ratio of recommended:non-recommended food items $(P<0 \cdot 0001)$ and unpolished:polished rice $(P<0 \cdot 0001)$.

\section{Discussion}

In this 5-year interventional trial in a young population at high risk of CVD, it has been shown that replacing white rice with unpolished rice may be a particularly effective individual dietary change to reduce risk factors for T2DM and CVD. Other foods and food groups that appear to exert their risk reduction effects via specific metabolic risk factors are also highlighted.

White rice consumption has been linked to T2DM risk in several cohort studies ${ }^{(21,22)}$. The relative risk is higher in Southern Asians per serving ${ }^{(22)}$ and Southern Asians consume more white rice than their Western counterparts ${ }^{(11,23)}$. Rice immediately harvested comprises the bran, germ and endosperm. White - or polished - rice is produced by milling which removes the hull, bran (primarily non-cellulosic polysaccharides, cellulose and lignin) and most of the wheatgerm $^{(24)}$. Unpolished rice retains beneficial fatty acids including oleic and linoleic $\operatorname{acid}^{(24)}$, protein, bran and some vitamins ${ }^{(24)}$ and is higher in fibre and has a lower glycaemic index than white rice ${ }^{(23,24)}$.

Total dietary fibre, cereal fibre and low glycaemic index foods have been repeatedly linked to improved glycaemic control and lower risk of T2DM in a variety of studies ${ }^{(25-29)}$. In general, whole grains, cereals or insoluble fibres are associated with lower T2DM risk in cohort studies ${ }^{(28)}$, whereas soluble fibres reduce postprandial glucose concentrations in the controlled setting ${ }^{(28)}$. Our data suggest that sources of whole-grain 

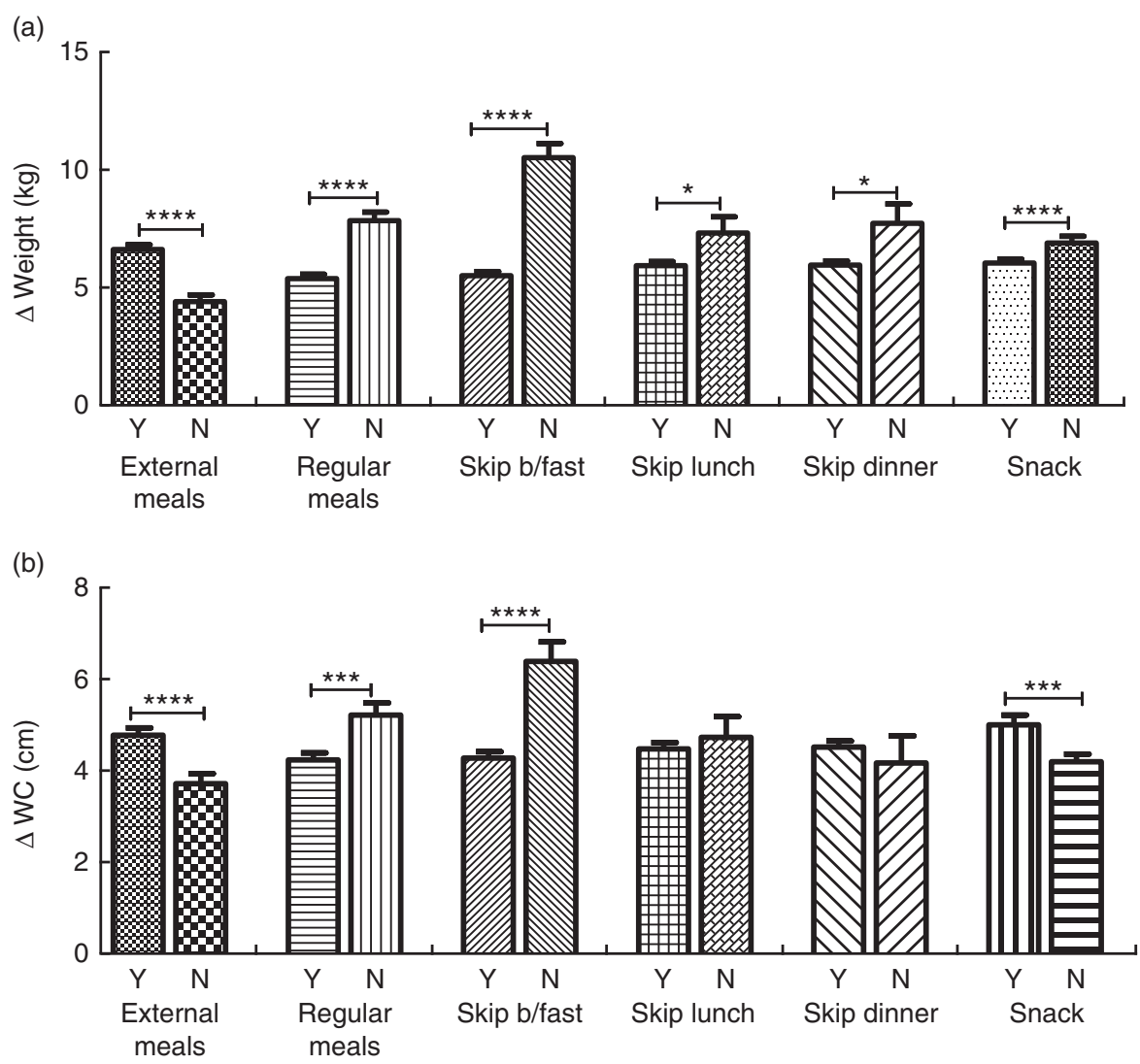

Fig. 1. Changes in weight (a) and waist circumference (b) at 5 years by whether or not meals are eaten out of the home (external meals), whether three regular meals are eaten per d (regular meals), whether breakfast (b/fast), lunch or dinner are delayed or skipped (skip b/fast, skip lunch, skip dinner) and whether $\geq 1$ snacks are consumed per d (Snacks). Differences determined by independent $t$ test. Values are means, with their standard errors represented by vertical bars. Snacks, regular snacks; N, no; WC, waist circumference; Y, yes. ${ }^{*} P<0.05,{ }^{\star \star \star} P<0.001,{ }^{\star \star \star \star} P<0.0001$.

Table 2. $\beta$-Coefficients for the effect of food patterns, groups and specific foods on fasting plasma glucose (FPG) and 2-h plasma glucose (2hPG)

\begin{tabular}{|c|c|c|c|c|}
\hline & \multicolumn{2}{|c|}{ FPG } & \multicolumn{2}{|c|}{ 2hPG } \\
\hline & $\beta$-Coefficient & $P$ & $\beta$-Coefficient & $P$ \\
\hline Recommended:non-recommended & -0.049 & 0.023 & -0.001 & 0.96 \\
\hline Unpolished:polished & -0.084 & 0.007 & 0.034 & 0.20 \\
\hline Soluble fibre & -0.003 & 0.91 & 0.009 & 0.63 \\
\hline Red meat & 0.050 & 0.018 & 0.001 & 0.961 \\
\hline Low-fat dairy products & -0.032 & 0.13 & -0.046 & 0.018 \\
\hline Regular meals & -0.049 & 0.01 & 0.014 & 0.46 \\
\hline Alcohol & -0.041 & 0.029 & -0.042 & 0.02 \\
\hline Physical activity (metabolic equivalents) & -0.005 & 0.77 & -0.037 & 0.038 \\
\hline
\end{tabular}

fibre (mainly insoluble) may exert their T2DM-preventive effects by specifically altering fasting glucose concentrations, as the association was strong and not altered by adjusting for multiple confounders, and no association was found between unpolished rice or starches on 2hPG concentrations in any model. There is limited epidemiological data on this question as the majority of cohort studies use T2DM incidence as the dependent variable, not FPG or 2hPG concentrations ${ }^{(25,26,29)}$, but controlled trials and cohort studies that measure FPG and 2hPG with appropriate adjustment have shown a reduction in fasting but not postprandial glucose $\mathrm{e}^{(30-32)}$. Fasting glucose concentrations are rising at a rate of $0.07 \mathrm{mmol} / 1$ per decade across the population ${ }^{(33)}$; our finding that replacement of white rice with unpolished rice significantly reduces FPG has important public health implications in countries such as Sri Lanka where rice is a staple food.

Dietary fibre has also been linked to reduced weight and WC in a number of cohort and controlled studies ${ }^{(34-36)}$, with the mechanism posited to be by a bulking effect, lowering energy density and/or the glycaemic index of the diet ${ }^{(28)}$. Products of 

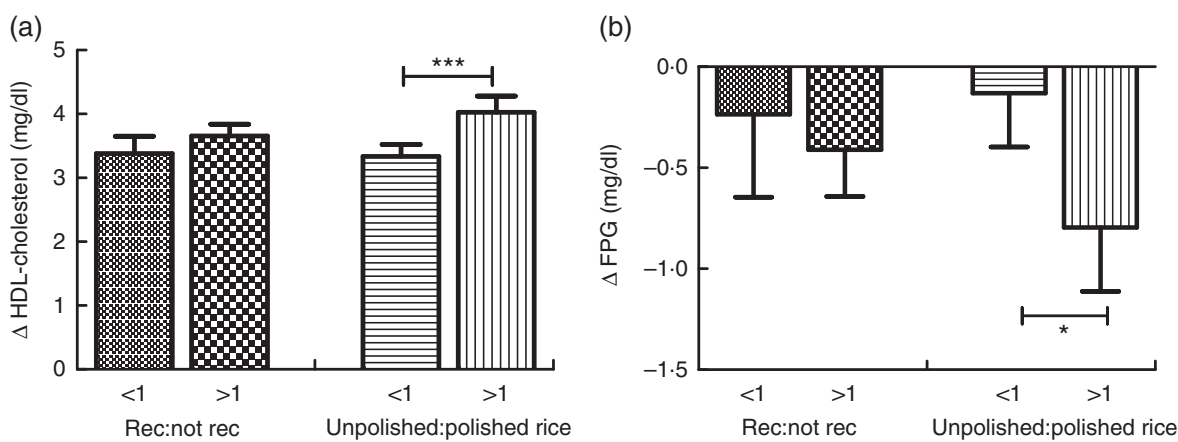

Fig. 2. Change in fasting plasma glucose (a) and HDL-cholesterol (b) among participants consuming a ratio of recommended:non-recommended items and a ratio of unpolished:polished rice of more or less than 1 . Differences determined by independent $t$ test. Values are means, with their standard errors represented by vertical bars. FPG, fasting plasma glucose; Rec, recommended. ${ }^{\star} P<0.05,{ }^{\star \star \star} P<0.001$.

Table 3. $\beta$-Coefficients for the effect of the ratio of unpolished rice:white rice on clinical and biochemical outcomes

\begin{tabular}{|c|c|c|c|c|}
\hline & \multicolumn{2}{|c|}{ HDL-cholesterol } & \multicolumn{2}{|c|}{ TAG } \\
\hline & $\beta$-Coefficient & $P$ & $\beta$-Coefficient & $P$ \\
\hline Recommended:non-recommended & 0.044 & 0.032 & -0.018 & 0.33 \\
\hline Unpolished:polished & 0.066 & 0.031 & -0.084 & 0.005 \\
\hline Soluble fibre & 0.28 & $0 \cdot 16$ & -0.044 & 0.025 \\
\hline Red meat & -0.009 & 0.65 & 0.034 & 0.083 \\
\hline Low-fat dairy products & -0.019 & 0.33 & 0.001 & 0.97 \\
\hline White fish & 0.037 & 0.06 & -0.059 & 0.003 \\
\hline Regular meals & 0.019 & 0.30 & 0.009 & 0.61 \\
\hline Alcohol & 0.035 & 0.06 & -0.025 & 0.21 \\
\hline Physical activity & -0.008 & 0.65 & -0.025 & 0.15 \\
\hline
\end{tabular}

dietary fibre fermentation have also been shown to increase the production of hormones, which decrease appetite ${ }^{(27,33,35)}$. For example, inulin supplementation attenuates weight gain in overweight children over 1 year ${ }^{(34)}$. Although it has been thought that soluble fibres are generally fermentable, while insoluble fibres are not ${ }^{(28)}$, other authors have shown that long-term, cereal fibres such as cellulose are fermented and associated with increases in glucagon-like peptide-1 (GLP-1) and a reduction in appetite ${ }^{(37)}$. As our regression models were adjusted for changes in weight, our data therefore suggest that substitution of white rice with unpolished rice has a twopronged effect on T2DM risk, first by limiting weight gain and second by its independent effects on FPG.

No effect was observed by replacing total high-fat food items with their low-fat varieties on any of the outcome measures studied. The role of fat in risk of T2DM and CVD is complex, with different fat sources, classes and separate fatty acids possibly having distinct and opposing effects on T2DM risk $^{(38)}$. Dairy fat in particular has been linked to a reduction in risk $^{(8,38)}$, although our finding showed that low-fat dairy products but not total dairy products were associated with a reduction in $2 \mathrm{hPG}$, suggesting that the erstwhile nutrients of dairy products such as $\mathrm{Ca}$ and milk proteins may be mediating this beneficial effect ${ }^{(39)}$.

An association between red meat and FPG was found, but not with 2hPG. This is supported by previous cohort studies ${ }^{(19)}$, with data also suggesting that red meat intake is specifically associated with fasting glucose and risk of impaired fasting glucose ${ }^{(40,41)}$. Although no association between processed meats and any outcome was found, in contrast to a body of work implicating processed meat in CHD and $\mathrm{T}_{2} \mathrm{DM}^{(42)}$, this may reflect the limited consumption of such foods in the Sri Lankan diet.

The lipid profile is an important component in the development of CVD, and Southern Asians have lower HDL-cholesterol compared with their Caucasian counterparts ${ }^{(2)}$. Although the relationship between whole grains, glucose concentrations and TAG levels are well documented ${ }^{(28)}$, there is little suggestion in the literature that dietary fibre influences HDL-cholesterol concentrations. In the present study, replacing white rice with unpolished rice was associated with increases in HDLcholesterol, and the relationship remained significant after adjusting for multiple factors including factors known to alter HDL-cholesterol such as alcohol intake, smoking status and physical activity. Interestingly, both consumption of white rice and glycaemic load of the diet negatively associate with HDL-cholesterol across a number of populations ${ }^{(43,44)}$. Therefore, it appears that adding whole grain to the diet is important for glycaemic control, weight management and reduction of TAG. In addition, its displacement of high glycaemic index, highly refined rice may be equally important for the benefit on HDL-cholesterol.

There is a growing body of data demonstrating the importance of meal timings on CVD risk factors ${ }^{(13,14)}$. Although our finding that regular meals are negatively associated with weight and WC is limited by the lack of control for energy intake, it is 
supported by data from large cross-sectional studies in the $\mathrm{UK}^{(13)}$ and Sweden ${ }^{(14)}$ and by prospective studies in children ${ }^{(45)}$ and adults ${ }^{(46)}$. After controlling for weight change, irregular meals was also positively associated with FPG, which may be due to the effect of irregular meals on circadian secretion of hormones including GLP-1 and insulin ${ }^{(46)}$. Advice to consume regular meals was easy to translate across this population and could be tested independently in a controlled setting.

Some interesting associations between dietary factors and specific components of the MetS were observed. It is now clear that the pre-diabetic period is characterised by two separate conditions - impaired fasting glucose and impaired glucose tolerance - each with its own distinct underlying pathophysiology ${ }^{(47)}$. Our findings highlight the distinct roles nutrients and meal patterning may play in the development of T2DM, and suggest that regular meals, a reduction in red meat and increased bran intake may specifically affect fasting glucose dysregulation, whereas physical activity and low-fat dairy products may target peripheral insulin resistance, which characterises impaired glucose tolerance ${ }^{(47)}$.

Some strengths of this study while acknowledging its limitations are highlighted. First, food intake data were collected every year during this 5-year study, enabling the calculation of average intake over time, and the reduction of withinperson measurement errors ${ }^{(23)}$. The long follow-up period also enabled the examination of the effect on clinical factors over time. However, because the FFQ - which themselves have limitations - were adapted to the target population, it was not validated. Although portion sizes were indicated by the participants using serving spoons, this method could have introduced error. The analyses cannot therefore be reliably adjusted for energy intake. Nevertheless, the primary findings of this study have been controlled for weight change over 5 years, which is arguably a more reliable estimate of energy intake and energy balance. In Sri Lankan cuisine, soya, sunflower, palm and coconut oils are used for cooking and have known effects on CVD risk factors ${ }^{(48,49)}$. Although fried foods were controlled for in our analyses, the FFQ did not include information on the different dietary oils or fats used in cooking and it is possible that differences in these sources mediated the relationships seen. This is particularly relevant as coconut oil is commonly consumed as an added factor in Sri Lanka, and is implicated in both promoting or limiting CVD progression ${ }^{(50,51)}$. We acknowledge our exchange model included some foods such as egg yolks and nuts in the non-recommended category, even though they are thought to have neutral or beneficial effects on T2DM or CVD risk in most people. However, its primary design was to reduce the energy density of the diet in a way that was simple to understand and translate for community-recruited educators who did not have a background in nutrition. Finally, these analyses used pooled data from the intervention and control group of a primary prevention trial. Thus, although the original study was controlled, this current analysis cannot infer causation, and the data were not adjusted for multiple comparisons.

In conclusion, a pragmatic dietary intervention can significantly reduce risk factors for the MetS in a high-risk population. Replacement of white with unpolished rice may be a particularly effective dietary advice for this and similar populations.

\section{Acknowledgements}

The authors acknowledge the contributions of Dr Thilinie Jayasekera, Talya Fernando, Dr Nuwan Ranawaka for their help with this work. The authors thank Dr Varsha, Dr Mahesh (MV Diabetes Centre, WHO collaborating centre for research education and training in diabetes, Chennai, India) and Dr Arambepola (University of Colombo Sri Lanka) for their advice and input as members of the DIABRISK-SL independent study advisory committee. The authors also wish to thank all the participants of the study.

This project is supported by a BRIDGES Grant from the International Diabetes Federation. BRIDGES, an International Diabetes Federation project, is supported by an educational grant from Lilly Diabetes and a grant awarded from the Diabetes Association of Sri Lanka. The funder had no role in the design, analysis or writing of this article.

J. K., G. V., L. G. and M. W. conceived the study and its design and were involved in implementation of the study. N. G. led the dietary analysis plan, carried out all data analysis and wrote the manuscript with feedback from the other authors. L. V., J. K., M. G., G. V., L. G. and M. W. were involved in coordination of the study. All the authors read and approved the final manuscript. N. G. is the guarantor of this work and, as such, had full access to all the data in the study and takes responsibility for the integrity of the data and the accuracy of the data analysis.

The authors declare no conflicts of interest.

\section{Supplementary material}

For supplementary material/s referred to in this article, please visit http://dx.doi.org/doi:10.1017/S0007114516002476

\section{References}

1. O'Neill S \& O'Driscoll L (2015) Metabolic syndrome: a closer look at the growing epidemic and its associated pathologies. Obes Rev 16, 1-12.

2. Pandit K, Goswami S, Ghosh S, et al. (2012) Metabolic syndrome in South Asians. Indian J Endocr Metab 16, 44-55.

3. Zivkovic AM, German JB \& Sanyal AJ (2007) Comparative review of diets for the metabolic syndrome: implications for nonalcoholic fatty liver disease. Am J Clin Nutr 86, 285-300 (Review).

4. Ambrosini GL, Emmett PM, Northstone K, et al. (2012) Identification of a dietary pattern prospectively associated with increased adiposity during childhood and adolescence. Int J Obes 36, 1299-1305.

5. Yamaoka K \& Tango T (2012) Effects of lifestyle modification on metabolic syndrome: a systematic review and metaanalysis. BMC Med 10, 138-148.

6. Kristensen M, Toubro S, Jensen MG, et al. (2012) Whole grain compared with refined wheat decreases the percentage of body fat following a 12-week, energy-restricted dietary intervention in postmenopausal women. J Nutr 142, 710-716.

7. Hollænder PL, Ross AB \& Kristensen M (2015) Whole-grain and blood lipid changes in apparently healthy adults: a systematic review and meta-analysis of randomized controlled studies. Am J Clin Nutr 102, 556-572. 
8. Louie JC, Flood VM, Rangan AM, et al. (2013) Higher regular fat dairy consumption is associated with lower incidence of metabolic syndrome but not type 2 diabetes. Nutr Metab Cardiovasc Dis 23, 816-821.

9. Babio N, Sorlí M, Bulló M, et al. (2012) Association between red meat consumption and metabolic syndrome in a Mediterranean population at high cardiovascular risk: cross-sectional and 1-year follow-up assessment. Nutr Metab Cardiovasc Dis 22, 200-207.

10. Radhika G, Sathya RM, Ganesan A, et al. (2011) Dietary profile of urban adult population in South India in the context of chronic disease epidemiology (CURES-68). Public Health Nutr 14, 591-598.

11. Misra A, Khurana L, Isharwal S, et al. (2009) South Asian diets and insulin resistance. BrJ Nutr 101, 465-473.

12. Shi Z, Taylor AW, Hu G, et al. (2012) Rice intake, weight change and risk of the metabolic syndrome development among Chinese adults: the Jiangsu Nutrition Study (JIN). Asia Pac J Clin Nutr 21, 35-43.

13. Pot GK, Hardy R \& Stephen AM (2014) Irregular consumption of energy intake in meals is associated with a higher cardiometabolic risk in adults of a British birth cohort. Int J Obes $\mathbf{3 8}$, 1518-1524.

14. Sierra-Johnson J, Undén AL, Linestrand M, et al. (2008) Eating meals irregularly: a novel environmental risk factor for the metabolic syndrome. Obesity 16, 1302-1307.

15. Wijesuriya M, Gulliford M, Vasantharajah L, et al. (2011) DIABRISK-SL prevention of cardio-metabolic disease with life style modification in young urban Sri Lankan's - study protocol for a randomized controlled trial. Trials 12, 209.

16. Ramachandran A, Snehalatha C, Mary S, et al. (2006) The Indian diabetes prevention programme shows that lifestyle modification and metformin prevent type 2 diabetes in Asian Indian subjects with impaired glucose tolerance (IDPP-1). Diabetologia 49, 289-297.

17. Twiss J, Dickinson J, Duma ST, et al. (2003) Community gardens: lessons learned from California healthy cities and communities. Am J Public Health 93, 1435-1438.

18. Dall'Alba V, Silva FM, Antonio JP, et al. (2013) Improvement of the metabolic syndrome profile by soluble fibre - guar gum - in patients with type 2 diabetes: a randomised clinical trial. Br J Nutr 110, 1601-1610.

19. Malik VS, Popkin BM, Bray GA, et al. (2010) Sugar-sweetened beverages and risk of metabolic syndrome and type 2 diabetes: a meta-analysis. Diabetes Care 33, 2477-2483.

20. Song Y, Manson JE, Buring JE, et al. (2004) A prospective study of red meat consumption and type 2 diabetes in middleaged and elderly women: the women's health study. Diabetes Care 27, 2108-2115.

21. Nanri A, Mizoue T, Noda M, et al. (2010) Japan public health center-based prospective study group. Rice intake and type 2 diabetes in Japanese men and women: the Japan public health center-based prospective study. Am J Clin Nutr 92, 1468-1477.

22. Sun Q, Spiegelman D, van Dam RM, et al. (2010) White rice, brown rice, and risk of type 2 diabetes in US men and women. Arch Intern Med 170, 961-969.

23. Hu EA, Pan A, Malik V, et al. (2012) White rice consumption and risk of type 2 diabetes: meta-analysis and systematic review. BMJ 344, e1454.

24. Slavin J (2003) Why whole grains are protective: biological mechanisms. Proc Nutr Soc 62, 129-134 (Review).

25. InterAct Consortium (2015) Dietary fibre and incidence of type 2 diabetes in eight European countries: the EPIC-InterAct study and meta-analysis of prospective studies. Diabetologia 58, 1394-1408
26. Schulze MB, Liu S, Rimm EB, et al. (2004) Glycemic index, glycemic load, and dietary fiber intake and incidence of type 2 diabetes in younger and middle-aged women. Am J Clin Nutr 80, 348-356.

27. Chandalia M, Garg A, Lutjohann D, et al. (2000) Beneficial effects of high dietary fiber intake in patients with type 2 diabetes mellitus. N Engl J Med 342, 1392-1398.

28. Weickert MO \& Pfeiffer AF (2008) Metabolic effects of dietary fiber consumption and prevention of diabetes. J Nutr $\mathbf{1 3 8}$, 439-442 (Review).

29. Schulze MB, Schulz M, Heidemann C, et al. (2007) Fiber and magnesium intake and incidence of type 2 diabetes: a prospective study and meta-analysis. Arch Intern Med 167, 956-965.

30. Karlström B, Vessby B, Asp NG, et al. (1984) Effects of an increased content of cereal fibre in the diet of type 2 (non-insulin-dependent) diabetic patients. Diabetologia 26, 272-277.

31. Lousley SE, Jones DB, Slaughter P, et al. (1984 May) High carbohydrate-high fibre diets in poorly controlled diabetes. Diabet Med 1, 21-25.

32. Bakker SJ, Hoogeveen EK, Nijpels G, et al. (1998) The association of dietary fibres with glucose tolerance is partly explained by concomitant intake of thiamine: the Hoorn Study. Diabetologia 41, 1168-1175.

33. Danaei G, Finucane MM, Lu Y, et al. (2011) National, regional, and global trends in fasting plasma glucose and diabetes prevalence since 1980: systematic analysis of health examination surveys and epidemiological studies with 370 country-years and $2 \cdot 7$ million participants. Lancet 378, 31-40.

34. Abrams SA, Griffin IJ, Hawthorne KM, et al. (2007) Effect of prebiotic supplementation and calcium intake on body mass index. J Pediatr 151, 293-298.

35. Ludwig DS, Pereira MA, Kroenke CH, et al. (1999) Dietary fiber, weight gain, and cardiovascular disease risk factors in young adults. JAMA 282, 1539-1546.

36. Liber A \& Szajewska H (2013) Effects of inulin-type fructans on appetite, energy intake, and body weight in children and adults: systematic review of randomized controlled trials. Ann Nutr Metab 63, 42-54.

37. Freeland KR, Wilson C \& Wolever TM (2010) Adaptation of colonic fermentation and glucagon-like peptide-1 secretion with increased wheat fibre intake for 1 year in hyperinsulinaemic human subjects. Br J Nutr 103, 82-90.

38. Morio B, Fardet A, Legrand P, et al. (2016) Involvement of dietary saturated fats, from all sources or of dairy origin only, in insulin resistance and type 2 diabetes. Nutr Rev 74, 33-47.

39. Pasin G \& Comerford KB (2015) Dairy foods and dairy proteins in the management of type 2 diabetes: a systematic review of the clinical evidence. Adv Nutr 6, 245-259.

40. Fretts AM, Follis JL, Nettleton JA, et al. (2015) Consumption of meat is associated with higher fasting glucose and insulin concentrations regardless of glucose and insulin genetic risk scores: a meta-analysis of 50,345 Caucasians. Am J Clin Nutr 102, 1266-1278.

41. Heikkilä HM, Schwab U, Krachler B, et al. (2012) Dietary associations with prediabetic states - the DR's EXTRA Study. Eur J Clin Nutr 66, 819-824.

42. Micha R, Michas G \& Mozaffarian D (2012) Unprocessed red and processed meats and risk of coronary artery disease and type 2 diabetes - an updated review of the evidence. Curr Atheroscler Rep 14, 515-524.

43. Radhika G, Ganesan A, Sathya RM, et al. (2009) Dietary carbohydrates, glycemic load and serum high-density lipoprotein cholesterol concentrations among South Indian adults. Eur J Clin Nutr 63, 413-420. 
44. Mosdøl A, Witte DR, Frost G, et al. (2007) Dietary glycemic index and glycemic load are associated with high-densitylipoprotein cholesterol at baseline but not with increased risk of diabetes in the Whitehall II Study. Am J Clin Nutr $\mathbf{8 6}$, 988-994.

45. Franko DL, Striegel-Moore RH, Thompson D, et al. (2008) The relationship between meal frequency and body mass index in black and white adolescent girls: more is less. Int J Obes $\mathbf{3 2}$, 23-29.

46. Pot GK, Hardy R \& Stephen AM (2016) Irregularity of energy intake at meals: prospective associations with the metabolic syndrome in adults of the 1946 British Birth Cohort. Br J Nutr 115, 315-323.

47. Abdul-Ghani MA, Tripathy D \& DeFronzo RA (2006) Contributions of beta-cell dysfunction and insulin resistance to the pathogenesis of impaired glucose tolerance and impaired fasting glucose. Diabetes Care 29, 1130-1139 (Review).

48. Medagama A, Fernando D \& Widanapathirana H (2015) Energy and nutrient intakes of Sri Lankan patients with type 2 diabetes mellitus: a cross-sectional survey. BMC Res Notes 8, 753.

49. Tennakoon SU, Kumar BN \& Meyer HE (2015) Differences in selected lifestyle risk factors for cardiovascular disease between Sri Lankans in Oslo, Norway, and in Kandy, Sri Lanka. Asia Pac J Public Health 27, NP616-NP625.

50. Eyres L, Eyres MF, Chisholm A, et al. (2016) Coconut oil consumption and cardiovascular risk factors in humans. Nutr Rev 74, 267-280.

51. Pietraszek A, Hermansen K, Pedersen SB, et al. (2013) Effects of a meal rich in medium-chain saturated fat on postprandial lipemia in relatives of type 2 diabetics. Nutrition 29, 1000-1006. 